Type I IFNs

(IFN-alfa \& IFN-beta)

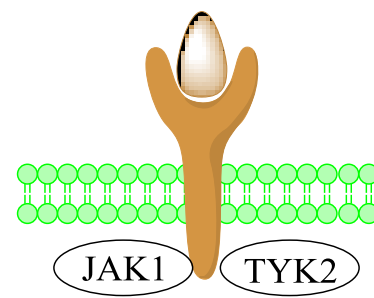

$\downarrow$

Antiviral response

Upregulation of

SARS-CoV-2

Receptor ACE2
IL-6

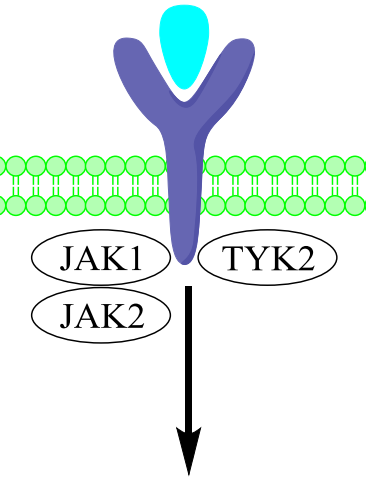

Acute phase responce

T-cell differentiation

Lymphocyte growth and differentiation

Lymphosyte effector Function
Type II IFNs

(IFN-gammaa)
IL-2, IL-4, IL-7

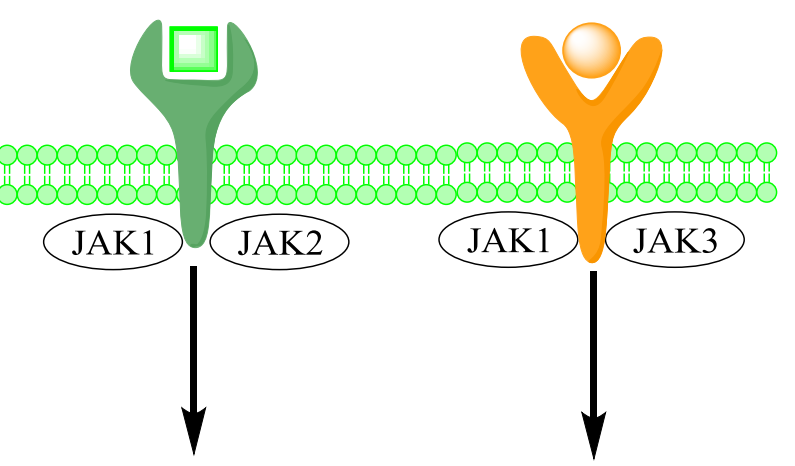

T-cell differentiation

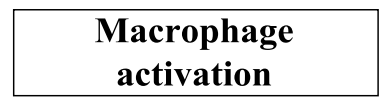

Lymphosyte effector Function

\begin{tabular}{|c|}
\hline $\begin{array}{c}\text { T-cell proliferation } \\
\text { \& survival }\end{array}$ \\
\hline T-cell memory \\
\hline $\begin{array}{c}\text { T-cell regulatory } \\
\text { function }\end{array}$ \\
\hline B-cell function \\
\hline
\end{tabular}

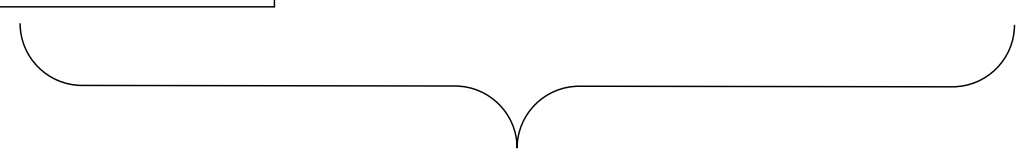

Dysregulated Immune responce

JAK-1 Selective Inhibitor 


\title{
The potential of JAK/STAT pathway inhibition as a New Treatment Strategy to Control Cytokine Release Syndrome in COVID-19
}

Mohammad Seyedhamzeh ${ }^{\mathrm{a}, \mathrm{b}}$, Hazha Omar Othman ${ }^{\mathrm{c}}$, Ali Aghajani Shahrivar ${ }^{\mathrm{d}}$, Fatemeh Aliabadi $^{\mathrm{b}}$, Mehdi Shafiee Ardestani ${ }^{{ }^{*}}$, Hamidreza Pazoki-Toroudi ${ }^{\mathrm{e}, \mathrm{b}^{*}}$

${ }^{a}$ Department of Radiopharmacy, Faculty of Pharmacy, Tehran University of Medical Sciences, Tehran, Iran.

${ }^{b}$ Physiology Research Center, Faculty of Medicine, Iran University of Medical Sciences, Tehran, Iran.

${ }^{c}$ Chemistry Department, College of Science, Salahaddin University, Erbil, Iraq.

${ }^{d}$ Chemistry Department, Central Tehran Branch, Islamic Azad University, Tehran, Iran

${ }^{e}$ Department of Physiology, Faculty of Medicine, Iran University of Medical Sciences, Tehran, Iran

Correspondence to: Hamidreza Pazoki-Toroudi

E-mail address: pazokihamidrezatoroudi95@gmail.com

\begin{abstract}
:
COVID-19, a pandemic affecting virus, which is caused by the current SARS-CoV2 coronavirus. The present research is performed on anti virus and immune-modulating therapies. Cytokine storms are the toxic drivers and mortality caused by various human viral infections. In addition, the intensity was linked to an elevated risk of acute respiratory failure, myocardial injury, and mortality in SARS-CoV-2-infected patients. The Janus kinase (JAK) therapeutic inhibitor class showed significant clinical benefits in anti-inflammatory and anti-viral effects. Among them, filgotinib has been approved as an active JAK inhibitor by decreasing biomarkers with main immune reaction functions and markers supporting matrix-degradation, angiogenesis, leukocyte adhesion, and recruitment in both research trials. In this study, we tried to get an insight into the choice of this drug in controlling the jack, to treat Covid 19 using drug design methods will be discussed.
\end{abstract}

\section{Keywords:}

COVID-19, JAK inhibitors, Filgotinib; cytokine release syndrome; immune modulation.

\section{Introduction:}

A new coronavirus disease with high mortality, emerging as pandemic disease, is the severe acute respiratory syndrome coronavirus 2 (SARS-CoV-2). Despite many public health initiatives, pharmacological therapies remain desperately required to treat patients affected, to decrease 
mortality, and to limit virus shedding and eventual transmission optimally. The infection with SARS-CoV-2 pushes the host to a deep cytokine response, which involves a sequence of mediators aimed at Immune-mediated inflammatory diseases (IMIDs). No specific therapy for COVID-19 is available up to now, cause many of the current treatments are symptomatic. (1) , (2)

Efficient prevention and care products are an immediate imperative, in particular in difficult, life-threatening situations. Pathogen infection with coronavirus (e.g., SARS, SARS-CoV-2) also contributes to the development of acute respiratory distress syndrome (ARDS) through severe cytokine and chemokine activity. Anti-viral cytokine signaling develops like a secondary haemophagocytic lymphohistiocytosis in some patients with moderate to severe COVID-19, hyper inflammatory status triggered by viral infections. Maintains the unacceptable levels of acute lung injury, chronic interferon activation, and deteriorate resistance to $\mathrm{T}$ cells and antibodies, perhaps for inadequate viral clearance. While the inflammation must mount most cytokines induced by SARS-CoV-2 infection and those attacked in some VIMIDs, they do not regulate the virus clearance. The role of immunosuppressive drugs widely used in immunemediated diseases in the susceptibility and natural history of COVID-19 can be appropriately taken and concern expressed. (3-5)

The cytokine excess associated with the SARS-CoV-2 reaction may also affect both viral clearance and defensive immune responses. Patients with autoimmune disorders have a high risk of infection as a result of endogenous and external factors such as immunosuppressants (dysfunctional immune system). One of the primary deficiencies of COVID-19 infection is the control of the cytokine storm. (6), (7)

As described above, current COVID-19 management is mostly positive, and medically validated therapies are not available. ARDS and cytokine storms are the leading causes of death. In addition, $50 \%$ of cytokine storm syndrome patients suffer from ARDS. Considering the exceptionally rapid progression of systemic and pulmonary inflammation in a subset of COVID19 patients, it is highly necessary to recognize and control the immune reactions that are disrupted at an early stage. However, this must be checked, and other biomarkers that are more sensitive and more precise can be identified. (8), (9)

Several explosive cytokines that include automatic diseases associated with their receptors have activated a JAK based phosphorylation cascade, which constitutes signals of gene transcription. Thus, medications block signals of cytokine that impede the action of JAK. These antagonists target medical treatment to HIV, RA, Psoriasis, Psoriatics, and inflammatory bowel diseases. (10)

Signal transduction plays a significant role in having and blocking the cytokine releases of the JAK family of enzymes and JAK inhibitors. Inhibitors of JAK can handle a cytokine storm by the induction of many inflammatory cytokines. Most inhibitors for JAK 1, JAK 2, less JAK 3, and the Tyrosine kinase 2 (TYK 2) are immensely successful for inhibition of Interleukin 6 (IL6) and interferon, but also inhibit the signal cascade of both Interleukin 2 (IL-2) and Interferon alfa or beta (IFN- $\alpha / \beta)$. JAK inhibitors have been efficient in inhibition. (11) 
Inhibitions of small molecules in JAK are quite a recent concept for systemic autoimmune/inflammatory conditions. JAK Inhibitors are biological inhibitors that interact with the Adenosine triphosphate (ATP) binding domain by inhibition of type I / II cytokine receptors. Jak inhibitors have provided targeted synthetic immunosuppressant products that interfere with JAK Signal transducer and activator of transcription (JAK-STAT) signaling by inhibiting one or more members of the JAK family (JAK1, JAK2, JAK3, TYK2). These molecules mediate the transcription factors of the STAT family, which contribute to pro-inflammatory cytokine release. Thus, cytokine expression can be decreased by them and help regulate cytokine storms. Additional inhibition of JAK by small modules can also be detrimental as they further restrict the isolation and clearance of the pathogen and can cause unexpected complications. (12)

On the other hand, these compounds are provided as medications orally, with highly trained pharmacodynamics and pharmacokinetics. They can provide a more practical approach to calm down the cytokine storm transiently to avoid ARDS and fulminating myocarditis. In addition to blocking IL-6, JAK1 inhibitors not only block inflammatory pathways in a cytokine storm. (13)

The aims of JAK1 and JAK3 affect some cytokines involved in anti-viral reactions such as interferons, IL-2, IL-15, IL-21, and IFN $\beta$. Thus, potentially, JAK1 inhibitors can inhibit SARSCoV-2 clearance. Inhibition of the SARS-CoV-2 or the IL-17-induced cytokine inhibits viral induction. In particular, it seems to be very promising to apply interleukin 6 (IL-6) and GM-CSF blockers to manage the massive cytokine storm that is linked to the development of lung damage typically and resulting ARDS in the most attacking patterns of SAR S-CoV disease. Both of whom rely on SARS-CoV-2 signaling in part or entirely (Figure 1). (14)

Therefore, clinical trials have been JAK1 inhibitors are also suggested as a safe treatment in hospitalized patients with COVID-19. Based on recent analyzes of the COVID-19 inflammatory markers and previous knowledge of inflammatory responses in other mortal lung infections, the potential strategy for anti-ARDS, brilliant myocarditis, organ failure, and mortality at an advanced stage of the condition has been assessed . (15)

One of the significant challenges in this regard is the replacement of more effective drugs. It has been indicated to be given to patients with COVID-19 in the late inflammatory process by baricitinib or other JAK inhibitors. Also, using different anti-viral medications, as a result of a growing understanding of infection pathophysiology, other drugs widely used in RA diagnosis have been proposed as alternative therapies for COVID-19. Baricitinib were tested for their anticytokine and anti-inflammatory function. Baricitinib induces cytokines with a lower IC50 value, which indicates that cytokine-induced JAK1 / STAT signalling becomes more impaired in the dosing period. It implies a more potent overall inhibition of cytokine-induced JAK1 / STAT signalling during dosing. But finding a drug with better therapeutic properties can help in the treatment of this disease. (16)

Finding new drugs is a challenging, expensive, and time-consuming task because there is no structured way to immediately discover a drug even though the drug activity's disorder, targets, and molecular mechanisms are well understood. There are millions of candidate molecules, and because of prohibitive costs, both in terms of time and energy, individual tests cannot be 
performed on any candidate. Reasonable drug design strategies have been introduced in recent months, particularly for In silico-based solutions, and this strategy has been backed by a recent study as a promising substitute or complementary method for efficient screening of potential drugs. Here, we used a bioinformatics approach to repurpose medication to classify the active antagonists of SARS-CoV2 Key Jak1-inhibitors. (17), (18)

\section{EXPERIMENTAL :}

In this study, the three-dimensional structures of ligand and proteins were obtained from PubChem and PDB database, respectively. Density functional theory at B3LYP/631+G (d, p) level implemented was used for 3D and geometry optimizations with energy minimization of each molecule. The protein-ligand interaction calculations were done by Autodock 4.2 and 2D ligand-protein interaction was calculated by Ligplot software. DRAGON software was used for molecular descriptors calculation. Genetic Algorithm and Partial least squares regression were used for feature selection. The evaluation of the active site, surface, and volume of protein was done by Computed Atlas for Surface Topography of Proteins (CASTp). Swiss ADME and target prediction were used to determine the pharmacokinetics properties and target analysis of molecules. GROMACS-2019 version using OPLS force field during was used for Molecular dynamic simulations during $20 \mathrm{~ns}$ by selecting periodic boundary conditions and the TIP3P water model for solvating complexes, followed by addition of ions to neutralize. Energy minimization was Tolerance for energy minimization was $1000 \mathrm{~kJ} / \mathrm{mol} / \mathrm{nm}$. (19-21)

\section{Results and discussion}

The interaction of the JAK1 inhibitor drugs (clinical and pre-clinical) using Autodock software was studied, and the results are given in Table 1.

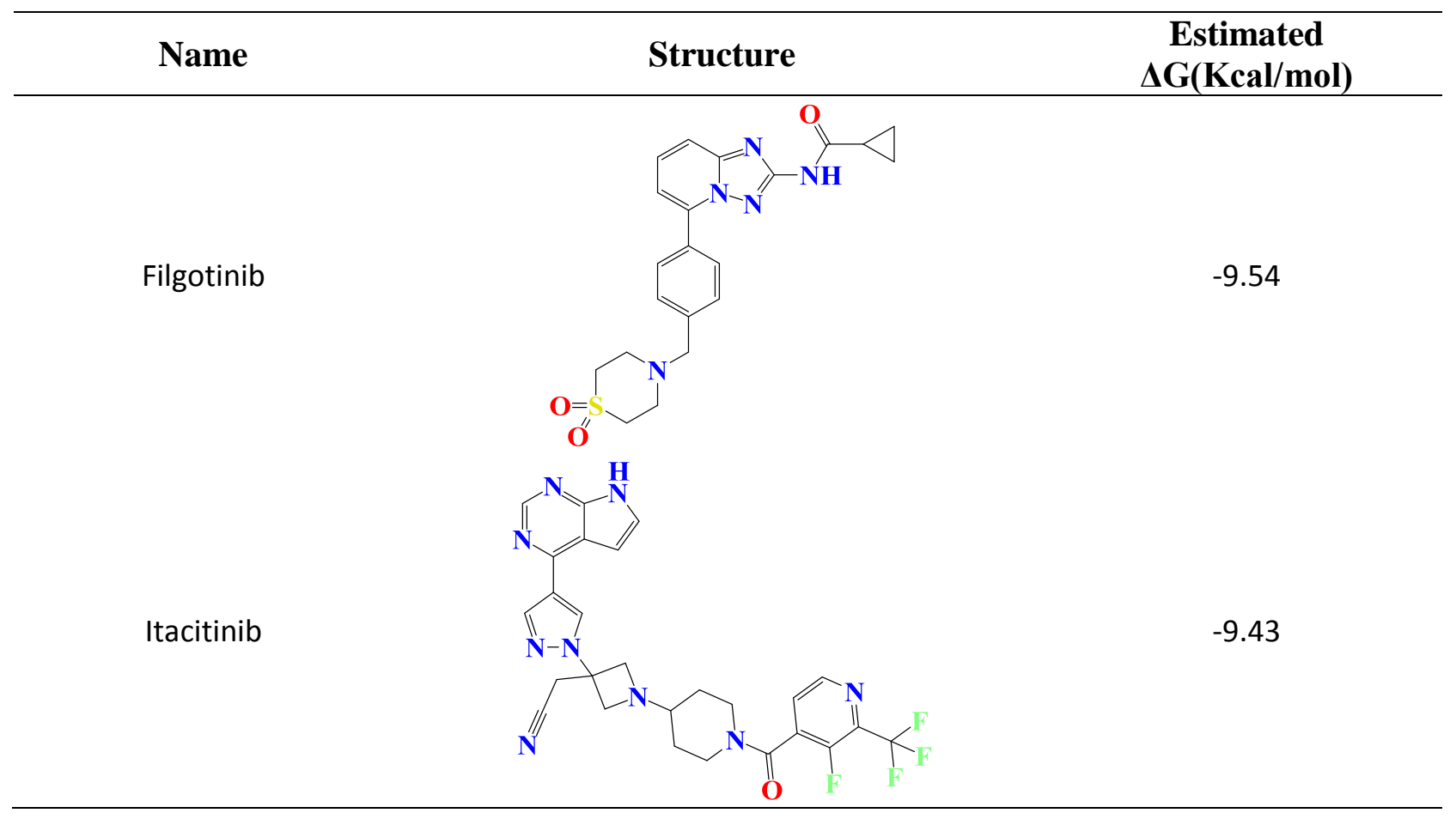




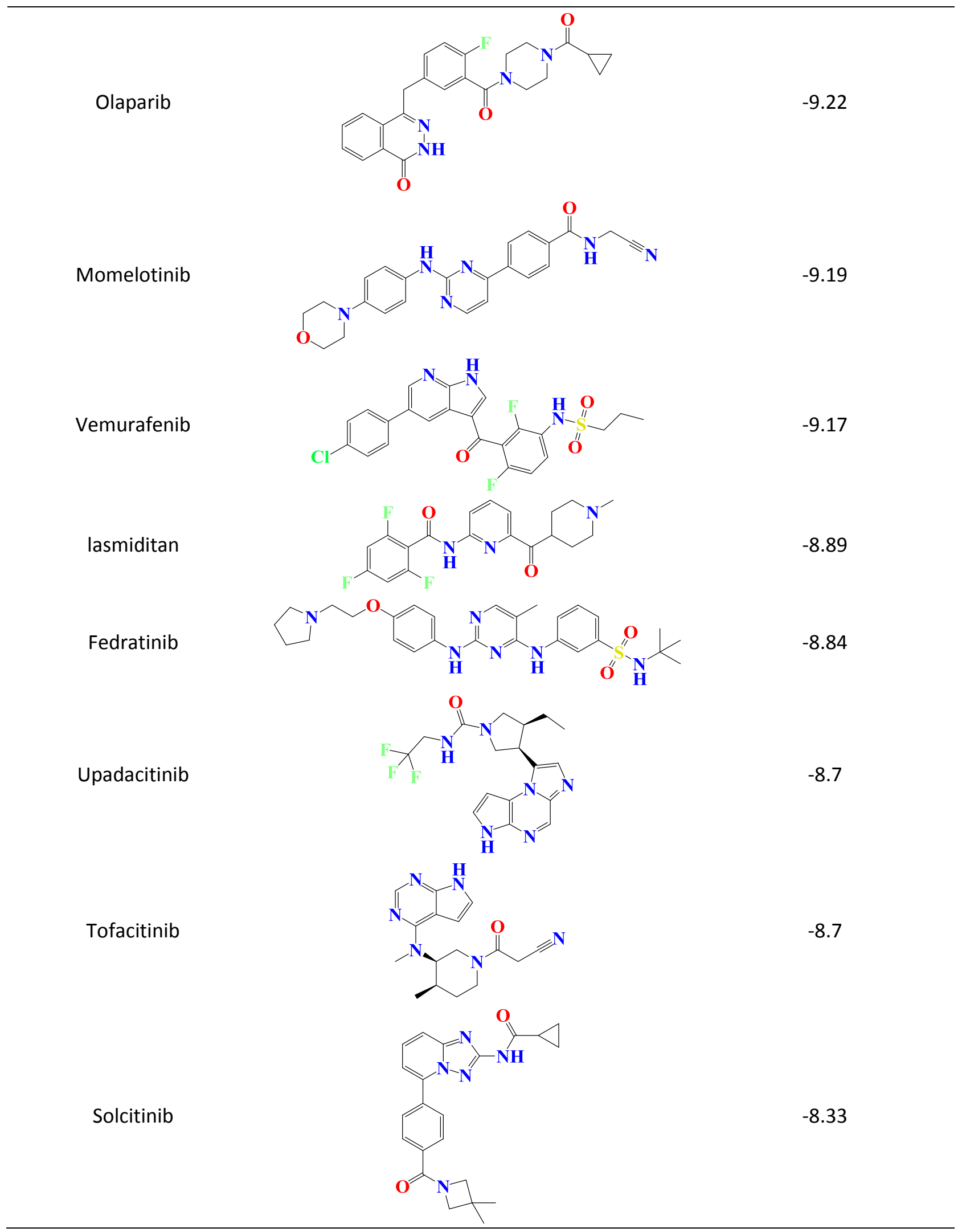


Azd-1480

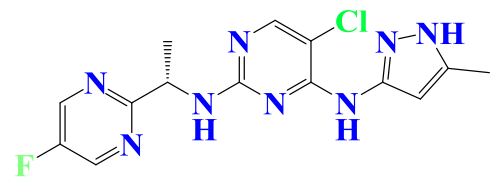

Baricitinib<smiles>c1nc(-c2cn[nH]c2)c2cc[nH]c2n1</smiles>

Semaxanib<smiles>CCS(=O)(=O)N1CC(C)(CC#N)C1</smiles>

Lasofoxifene<smiles>Oc1ccc2c(c1)CC[C@H](c1ccccc1)[C@H]2c1ccc(OCCN2CCCC2)cc1</smiles>

Mirogabalin

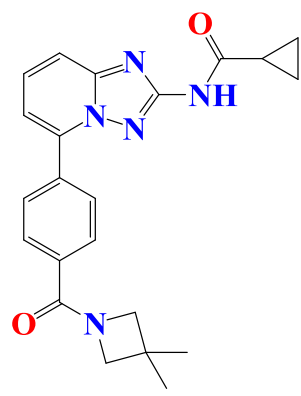

Fadrozole

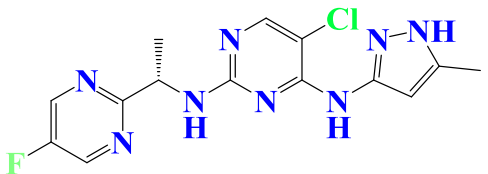

The binding force of molecular docking demonstrates the affinity of a specific ligand and energy, by which a compound interacts and binds to the pocket of a target protein. As a potential drug choice, a compound with fewer binding energy is favoured. The results showed that the drug Filgotinib is more stable with Jack1.

Filgotinib (GLPG0634 / GS-6034) is an active and selective inhibitor of JAK1 that under investigation in the treatment of RAs and inflammatory bowel disease. Filgotinib has demonstrated promising efficacy and is well tolerated for the treatment of rheumatoid arthritis. It 
is an orally delivered, potent, and selective seed inhibitor of JAK1. The pharmacokinetics and active metabolite of filgotinib in safe volunteers and the usage and analysis of pharmacokineticpharmaceutical models to help the design of the dosage for Phase IIB for patients with rheumatoid arthritis were addressed here. Two-phase II tests of another treatment for JAK1filgotinib in 2018 showed effectiveness in both patients with psoriatic arthritis 1 and ankylosing spondylitis in patients2. Compared to Upadacitinib 3,4, Filgotinib therapy provided a mean positive improvement in hemoglobin and platelet counts.

A study of the interaction of 33 derivatives of this drug using Autodock software showed that compared to the drugs studied in the previous section, $\Delta \mathrm{G}$ shifted to more stable values (Table 2) (Figure -2). QSAR calculations performed using algorithm-PLS genetics showed that the number of benzene ring and polarity is an essential factor in molecule-JAK1 interaction (Figure-2). Also, compared to filgotinib, only entry five has created a more stable complex. Pharmacophore analysis showed that the behaviour of this substance is similar to that of filgotinib, and changes in volume and area are similar (Table-3) (Figure 4) (Figure-5). But the results of 2d interaction result showed more hydrophobic interaction with amino acids (Table-4) (Figure-6). Target prediction results show that the A 93\% composition targets the LAK, while the B 73.3\% composition interacts (Figure-7). ADME studies also showed similar behavioural similarities to filgotinib (Table-5). As a result of these two compounds can be the alternative of Barticinib drug.

Table 2. Estimated $\Delta \mathrm{G}$ for filgotinib derivatives.

\begin{tabular}{|l|l|l|l|}
\hline & Measured & Predicted & Type \\
\hline
\end{tabular}




\begin{tabular}{|l|l|l|}
\hline \\
\hline
\end{tabular}




\begin{tabular}{|l|l|l|}
\hline & \\
\hline
\end{tabular}




\begin{tabular}{|l|l|l|}
\hline & \\
\hline
\end{tabular}




\begin{tabular}{|l|l|l|}
\hline \\
\hline
\end{tabular}




\begin{tabular}{|l|l|l|}
\hline & \\
\hline
\end{tabular}




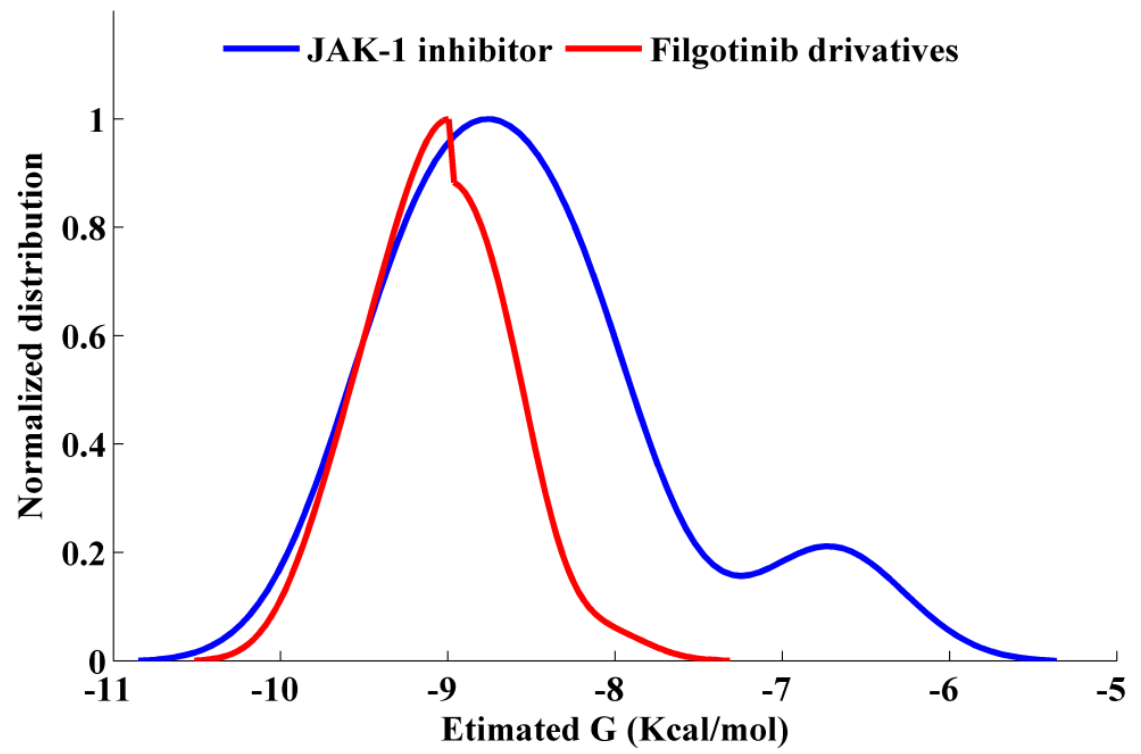

Figure 2. Estimated $\Delta \mathrm{G}$ distribution filgotinib derivatives (red) and JAK-1 inhibitors drug (blue)

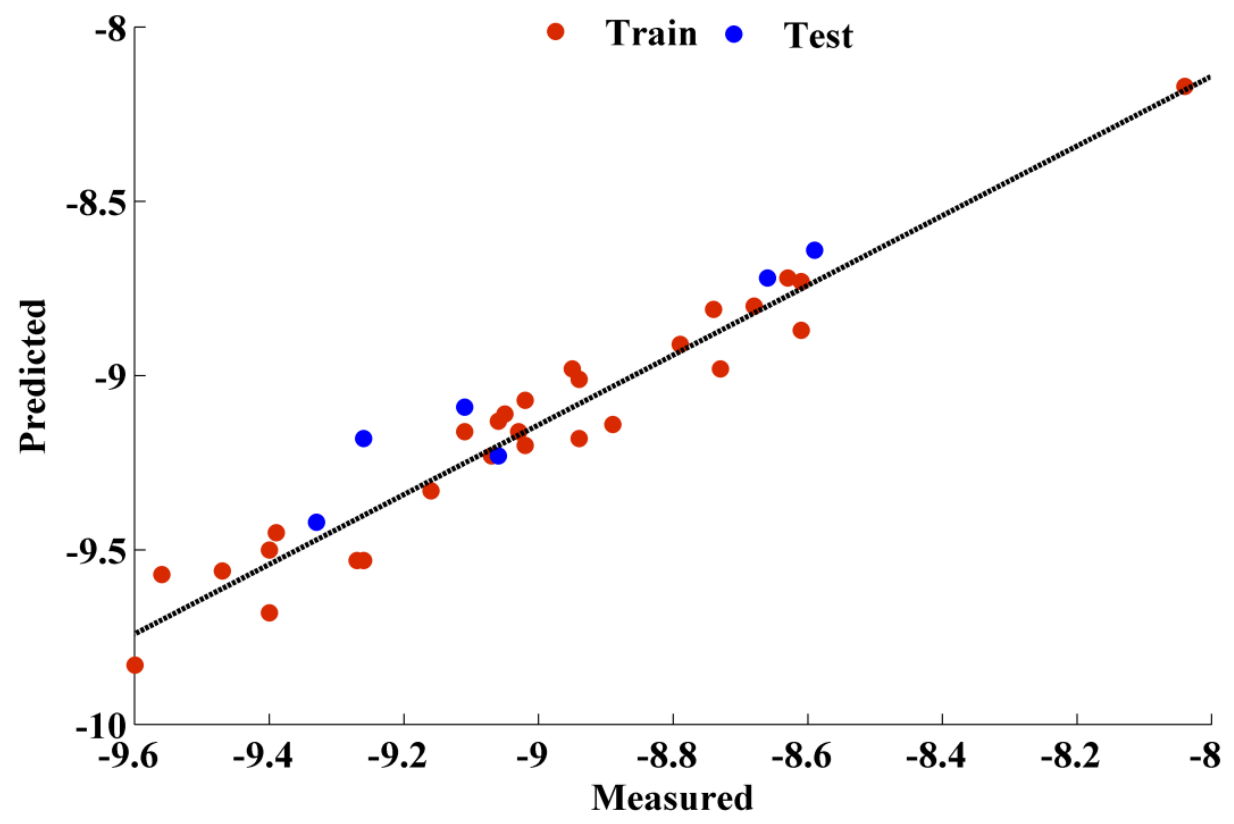

Figure 3. Measured and predicted results obtained from QSAR study 


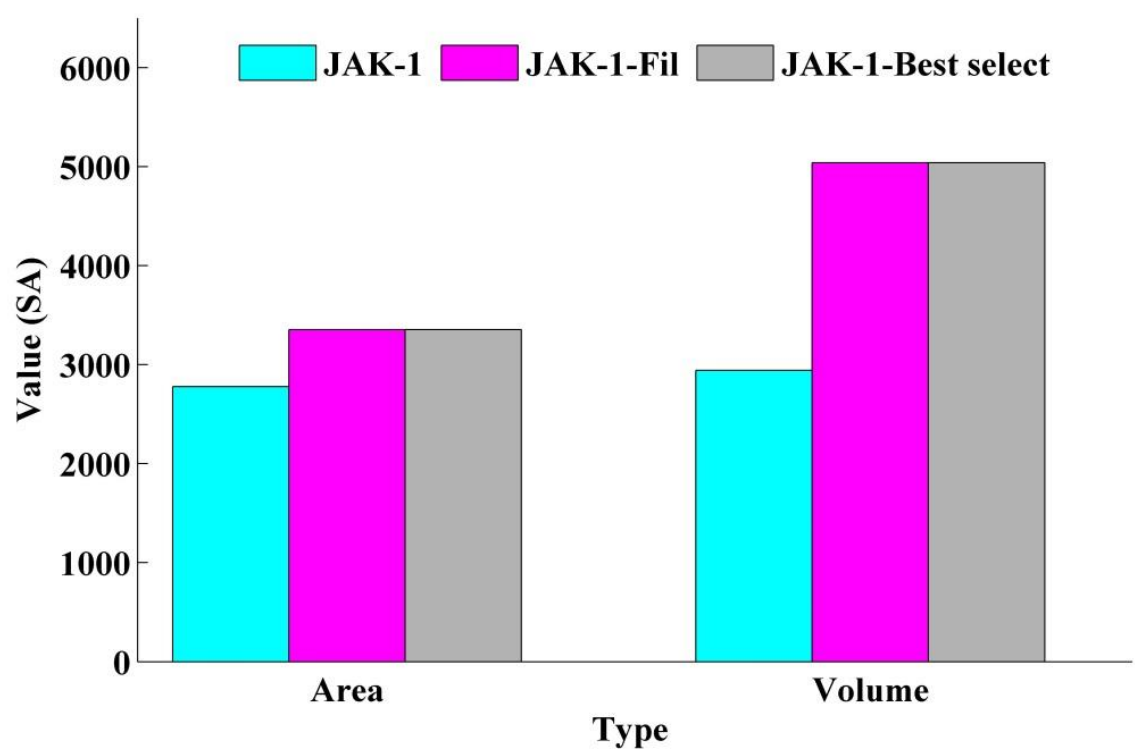

Figure 4._Comparison of the area and volume for Filgotinib-JAK-1 (pink) and Best selectedJAK-1 (grey) complexes with JAK-1 (cyan)

(a)

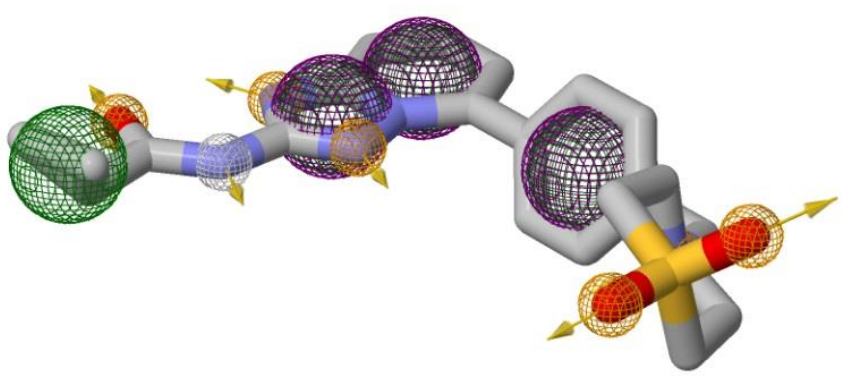

(b)

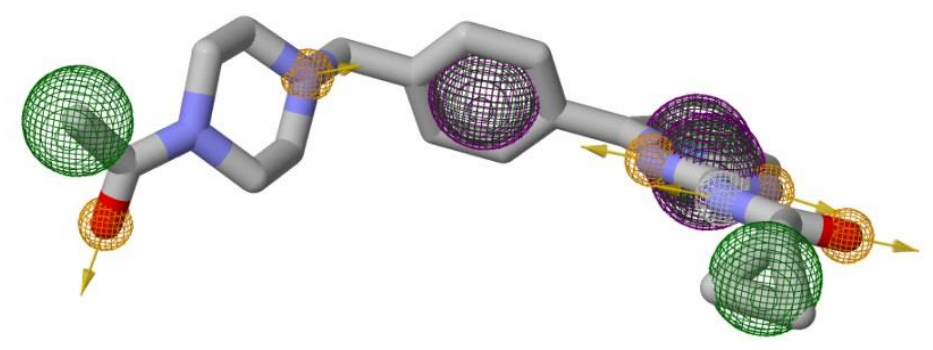


Figure 5. Pharmacophore study of (a) Filgotinib and (b) Best selected

Table 3. Results obtained from pharmacophore study of Filgotinib and Best selected

\begin{tabular}{|c|c|c|c|c|c|c|c|c|}
\cline { 2 - 9 } \multicolumn{1}{c|}{} & \multicolumn{4}{c|}{ Filgotinib } & \multicolumn{4}{c|}{$\mathrm{C}_{23} \mathrm{H}_{26} \mathrm{~N}_{6} \mathrm{O}_{2}$} \\
\hline Pharmacophore & $\mathrm{x}$ & $\mathrm{y}$ & $\mathrm{z}$ & Radius & $\mathrm{x}$ & $\mathrm{y}$ & $\mathrm{z}$ & Radius \\
\hline Aromatic & 3.4 & 2.56 & -0.38 & 1.1 & 2.9 & -2.71 & 0.3 & 1.1 \\
\hline Aromatic & -0.83 & 2.25 & 0.24 & 1.1 & -1.3 & -2.04 & -0.23 & 1.1 \\
\hline Aromatic & 3.63 & 0.5 & -0.03 & 1.1 & 3.29 & -0.67 & 0 & 1.1 \\
\hline HydrogenAcceptor & -4.14 & 0.64 & 0.59 & 0.5 & 3.75 & 1.72 & -0.34 & 0.5 \\
\hline HydrogenAcceptor & 2.54 & 0.05 & 0.18 & 0.5 & -4.49 & -0.17 & -0.48 & 0.5 \\
\hline HydrogenAcceptor & 4.79 & 0.25 & -0.13 & 0.5 & 2.23 & -0.12 & -0.19 & 0.5 \\
\hline HydrogenAcceptor & 6.24 & -2.21 & 0.13 & 0.5 & 4.47 & -0.51 & 0.08 & 0.5 \\
\hline HydrogenAcceptor & -7.26 & -2.26 & -1.87 & 0.5 & 6.1 & 1.84 & -0.14 & 0.5 \\
\hline HydrogenDonor & 3.91 & -1.9 & 0.36 & 0.5 & -6.09 & 3.74 & 0.28 & 0.5 \\
\hline Hydrophobic & 3.4 & 2.56 & -0.38 & 1 & -7.28 & 1.7 & 1.15 & 0.5 \\
\hline Hydrophobic & -0.83 & 2.25 & 0.24 & 1 & 2.9 & -2.71 & 0.3 & 1 \\
\hline Hydrophobic & 3.63 & 0.5 & -0.03 & 1 & -1.3 & -2.04 & -0.23 & 1 \\
\hline Hydrophobic & -7.85 & -2.99 & 0.3 & 1 & 3.29 & -0.67 & 0 & 1 \\
\hline Hydrophobic & 4.8 & -4.7 & 0.01 & 1 & 4.86 & 4.43 & 0.06 & 1 \\
\hline
\end{tabular}

(a)

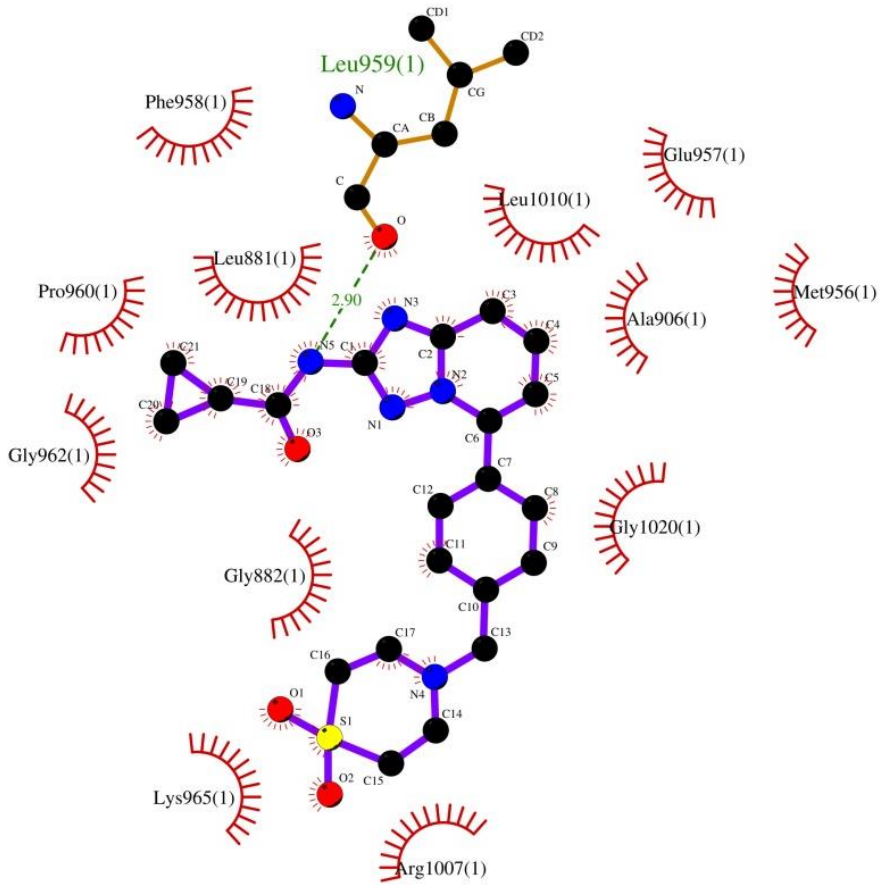

(b) 


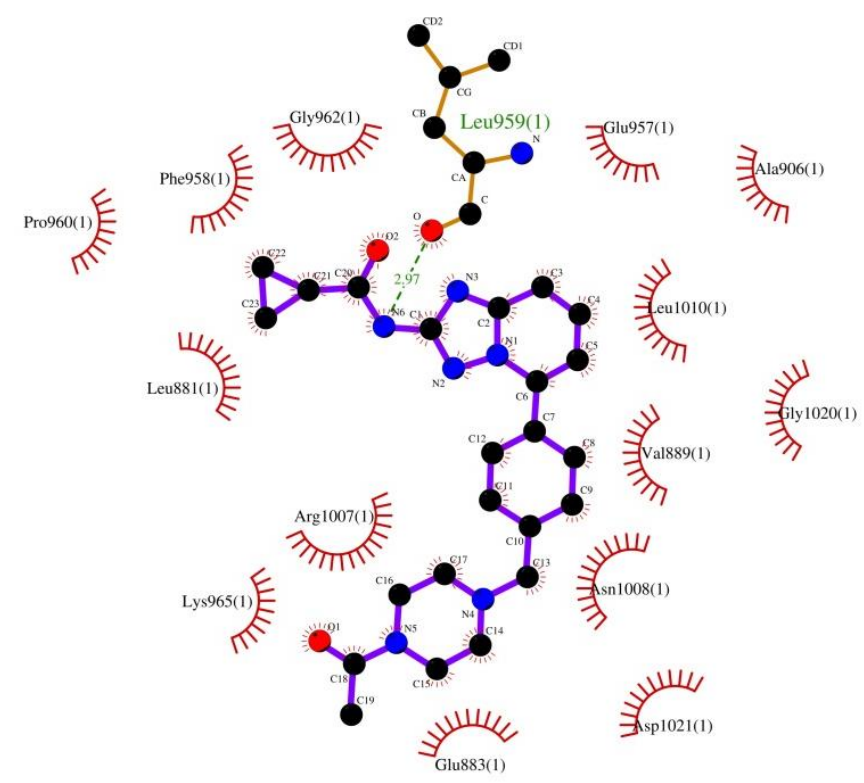

Figure 6. 2d interaction of (a) Filgotinib-JAK-1 and (b) Best selected-JAK-1

Table 4. Comparison of hydrogen and hydrophobic bond obtained from Filgotinib-JAK-1 and Best selected-JAK-1 interaction.

\begin{tabular}{|c|c|c|}
\hline Drug & Hydrogen Bond & Hydrophobic Bond \\
\hline Filgotinib & Leu959 & $\begin{array}{r}\text { Leu881,Gly882,Pro960,Gly962,Lys965,Phe958,Ala906, } \\
\text { Met956,Gly1020,Glu957,Leu1010 }\end{array}$ \\
\hline $\mathrm{C}_{23} \mathrm{H}_{26} \mathrm{~N}_{6} \mathrm{O}_{2}$ & Leu959 & $\begin{array}{c}\text { Leu881,Phe958,Pro960,Gly962,Lys965,Arg1007,Glu883, } \\
\text { Asp1021,Asn1008,Val889,Gly1020,Leu1010,Ala906,Glu } \\
\text { 957 }\end{array}$ \\
\hline
\end{tabular}

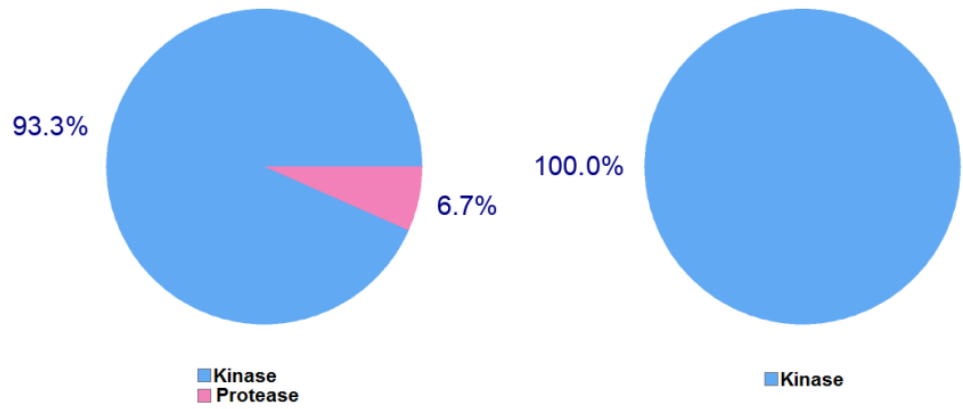

Figure 7. Target analysis of best selected (left) and filgotinib (right) 
Table 5. ADME study of best selected and filgotinib

\begin{tabular}{|c|c|c|c|c|c|}
\hline \multicolumn{3}{|c|}{ Pharmacokinetics } & \multicolumn{3}{|c|}{ Druglikeness } \\
\hline Parameter & Filgotinib & $\begin{array}{c}\text { best } \\
\text { selected }\end{array}$ & Parameter & Filgotinib & $\begin{array}{c}\text { best } \\
\text { selected }\end{array}$ \\
\hline GI absorption & High & High & Lipinski & Yes & Yes \\
\hline BBB permeant & No & No & Ghose & Yes & Yes \\
\hline P-GP substrate & Yes & Yes & Veber & Yes & Yes \\
\hline CYP1A2 inhibitor & No & No & Egan & Yes & Yes \\
\hline CYP2C19 inhibitor & No & No & Muegge & Yes & Yes \\
\hline CYP2C9 inhibitor & Yes & Yes & \multirow{4}{*}{$\begin{array}{l}\text { Bioavailability } \\
\text { Score }\end{array}$} & \multirow{4}{*}{0.55} & \multirow{4}{*}{0.55} \\
\hline CYP2D6 inhibitor & Yes & No & & & \\
\hline CYP3A4 inhibitor & Yes & Yes & & & \\
\hline Log Kp (skin permeation) & $-7.80 \mathrm{~cm} / \mathrm{s}$ & $-7.93 \mathrm{~cm} / \mathrm{s}$ & & & \\
\hline
\end{tabular}

The results of molecular dynamics studies of Root mean square deviation (RMSD), Root means, and Radius of gyration values are shown in Figure 8. The RSMD results, after $1.8 \mathrm{~ns}$, the structure reached stability were the mean values for entry five was $0.37 \mathrm{~nm}$. The RSMF results of these compounds represent less fluctuation with the average values of $0.34 \mathrm{~nm}$.

(a)

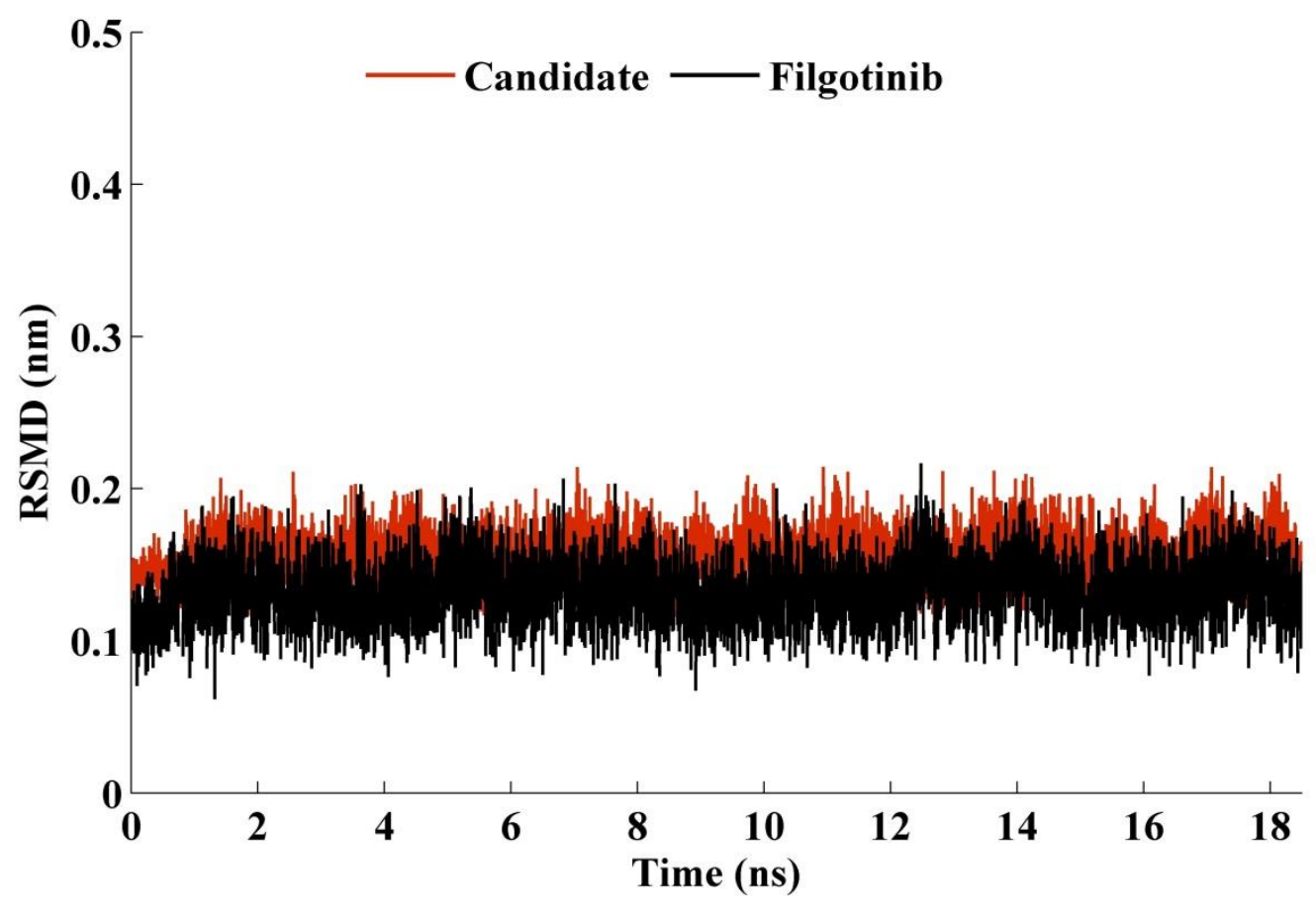

(b) 


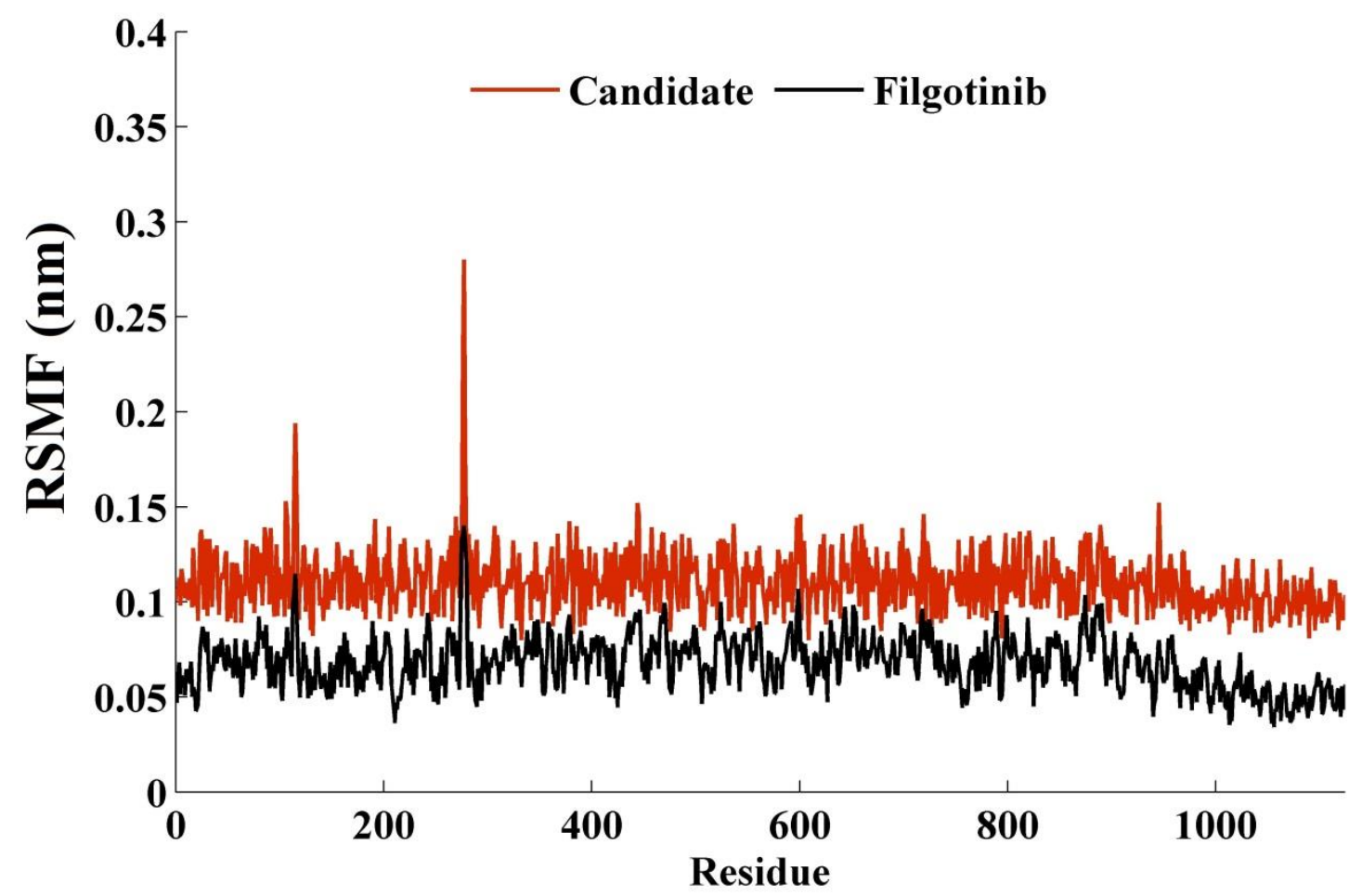

Figure 8. (a) Root mean square deviation (RMSD), (b) Root mean square fluctuation (RMSF) values for Entry 5

\section{Results:}

Due to the lack of a definitive drug to treat COVID-19, Selective inhibition of JAK-1 is one of the suggested treatments. The obtained results from this study suggest new alternatives to treat JAK1 kinase may reduce livery pathology, blocks both the human immune phenotype and related cytokine wind. These results have possible far-reaching importance to treat COVID-19. Still, need supports a more in-depth study on JAK-1 inhibits as the mechanism for therapeutic prevention of a cytokine storm and the downstream organ failure under this situation.

\section{Compliance with ethical guidelines Conflict of interest:}

The Autors have no financial or non-financial conflict of interest to declare. For this article, no studies with human participants or animals were performed by any of the authors. All studies conducted were in accordance with the ethical standards indicated in each case.

\section{References:}

(1): $\mathrm{Wu}, \mathrm{F}$. et al. A new coronavirus associated with human respiratory disease in China. Nature 579, 265-269 (2020). 
(2): COVID-19: risk for cytokine targeting in chronic inflammatory diseases? Georg Schett1,2 $\square$, Michael Sticherling2,3 and Markus F. Neurath2,4 272 | may 2020|volume 20

(3): Kugathasan, S., et al., Prediction of complicated disease course for children newly diagnosed with Crohn' s disease: a multicentre inception cohort study. The Lancet, 2017. 389(10080): p. 1710-1718.

(4): Hoffmann, M., et al., SARS-CoV-2 Cell Entry Depends on ACE2 and TMPRSS2 and Is Blocked by a Clinically Proven Protease Inhibitor. Cell, 2020: p. 1-19.

(5): Gordon, D.E., et al., A SARS-CoV-2-Human Protein-Protein Interaction Map Reveals Drug Targets and Potential Drug-Repurposing. bioRxiv, 2020.

(6): E.G. Favalli, F. Ingegnoli, O. De Lucia, et al., COVID-19 infection and rheumatoid arthritis: Faraway, so close!, Autoimmunity Reviews (2020), https://doi.org/10.1016/j.autrev.2020.102523

(7): Cytokine Release Syndrome in COVID-19 Patients, A New Scenario for an Old Concern: The Fragile Balance between Infections and Autoimmunity Andrea Picchianti Diamanti 1,* , Maria Manuela Rosado 2, Claudio Pioli 3 , Giorgio Sesti 1 and Bruno Laganà, International Journal of Molecular Sciences, Int. J. Mol. Sci. 2020, 21, 3330; doi:10.3390/ijms21093330

(8): C. Huang, Y. Wang, X. Li, L. Ren, J. Zhao, Y. Hu, et al., Clinical features of patients infected with 2019 novel coronavirus in Wuhan, China. Lancet. 395 (10223) (2020) 497-506

(9); Y. Du, L. Tu, P. Zhu, M. Mu, R. Wang, P. Yang, et al., Clinical Features of 85 Fatal Cases of COVID-19 from Wuhan: A Retrospective Observational Study, Am. J. Respir. Crit. Care Med. (2020), https://doi.org/10.1164/rccm.202003-0543OC.

(10): Gao J, Tian Z, Yang X. Breakthrough: Chloroquine phosphate has shown apparent efficacy in treatment of COVID-19 associated pneumonia in clinical studies. Biosci Trends 2020:2020.01047. doi:10.5582/bst.2020.01047.

(11): COVID-19: Immunology and treatment options Susanna Felsensteina, Jenny A. Herbertb, Paul S. McNamarab, Christian M. Hedrichb,c, $\square$ Clinical Immunology 215 (2020) 108448, https://doi.org/10.1016/j.clim.2020.108448.

(12): Selective Janus kinase inhibitors come of age John J. O' Shea and Massimo Gadina, Nature reviews | RheumAtology, https://doi.org/10.1038/s41584-018-0155-9

(13): Burmester, G. R. et al. Safety and efficacy of upadacitinib in patients with rheumatoid arthritis and inadequate response to conventional synthetic disease-modifying anti-rheumatic drugs (SELECT-NEXT): a randomised, double-blind, placebo-controlled phase 3 trial. Lancet 391, 2503-2512 (2018). 
(14): . Nakase T, Wada H, Minamikawa K et al (1994) Increased activated protein C-protein C inhibitor complex level in patients positive for lupus anticoagulant. BloodCoagul Fibrinolysis5:173-177

(15): Relative efficacy and safety of tofacitinib, baricitinib, upadacitinib, and filgotinib in comparison to adalimumab in patients with active rheumatoid arthritis, Zeitschrift für Rheumatologie, Young Ho Lee · Gwan Gyu Song, Nature 2020, https://doi.org/10.1007/s00393020-00750-1

(16): Genovese, M. C. et al. Safety and efficacy of upadacitinib in patients with active rheumatoid arthritis refractory to biologic disease-modifying anti-rheumatic drugs (SELECTBEYOND): a doubleblind, randomised controlled phase 3 trial. Lancet 391, 2513-2524 (2018).

(17): Combe B, Kivitz A, Tanaka Y, van der Heijde D, Matzkies F, Bartok B, Ye L, Guo Y, Tasset C, Sundy J (2019) Efficacy and safety of filgotinib for patients with rheumatoid arthritis with inadequate response to methotrexate: FINCH1 primaryoutcomeresults

(18): Gadina, M. et al. Translational and clinical advances in JAK-STAT biology: the present and future of jakinibs. J. Leukoc. Biol. 104, 499-514 (2018).

(19): Current Topics in Medicinal Chemistry, 2008, 8, 1628-1655 1568-0266/08 \$55.00+.00 () 2008 Bentham Science Publishers Ltd. Applications of 2D Descriptors in Drug Design: A DRAGON Tale Aliuska Morales Helgueraa,b,c, Robert D. Combesd, Maykel Pérez Gonzálezc, $\dagger$ and M. Natália D.S. Cordeiroa,*

(20): DRAGON SOFTWARE: AN EASY APPROACH TO MOLECULAR DESCRIPTOR CALCULATIONS Andrea Mauri*, Viviana Consonni, Manuela Pavan, and Roberto Todeschini, MATCH Commun. Math. Comput. Chem. 56 (2006) 237-248.

(21): Hybrid-Genetic Algorithm based Descriptor Optimization and QSAR Models for Predicting the Biological Activity of Tipranavir Analogs for HIV Protease Inhibition A. Srinivas Reddy1, ${ }^{*}$, Sunil Kumar2, and Rajni Garg. J Mol Graph Model. 2010 June ; 28(8): 852-862. doi:10.1016/j.jmgm.2010.03.005. 